\title{
VALORACIÓN DEL ESTADO DE SALUD Y PSICOPATOLOGÍA DE LOS FAMILIARES EN EL TRASTORNO DEL COMPORTAMIENTO ALIMENTARIO: DIFERENCIAS ENTRE CUIDADORES PRINCIPALES Y SECUNDARIOS
}

\author{
Enrique Berbel ${ }^{1}$, Ana R. Sepúlveda ${ }^{1}$, Monserrat Graell ${ }^{2}$, Patricia Andres ${ }^{2}$, \\ JOSÉ A. CARROBLES ${ }^{1}$ Y GONZALO MORANDÉ ${ }^{2}$ \\ ${ }^{1}$ Facultad de Psicología, Universidad Autónoma, Madrid \\ ${ }^{2}$ Unidad de Trastornos Alimentarios, Hospital Universitario Infantil Niño Jesús, Madrid
}

\begin{abstract}
Resumen: El objetivo del estudio fue explorar las diferencias en el estado general de salud y psicopatología entre cuidadores principales y secundarios de pacientes con trastorno del comportamiento alimentario. Se suministró una batería de cuestionarios a una muestra de 186 familiares ( 93 cuidadores principales y 93 secundarios). Los resultados indicaron que los cuidadores principales presentaban peores valores en malestar psicológico, ansiedad, depresión, y síntomas psicopatológicos en comparación con el grupo de cuidadores secundarios. La frecuencia de sujetos (cuidadores primarios versus secundarios) que alcanzaron el punto de corte de gravedad psicopatológica (GSI del SCL-90-R) fue similar para ambos grupos. Aunque los dos grupos de cuidadores presentaban una prevalencia de psicopatología similar, los resultados sugieren que sobre todo los cuidadores primarios se encuentran en situación de riesgo para su salud mental general, ansiedad y depresión.
\end{abstract}

Palabras clave: cuidador principal, trastorno alimentario, experiencia negativa, ansiedad, depresión, psicopatología.

\section{Assessment of general health and psychopathology among family members in eating disorder settings: Differences between primary and secondary caregivers}

\begin{abstract}
The purpose of this work was to assess differences in general health and psychopathology between primary and secondary caregivers of patients with a diagnosis of eating disorder. A battery of questionnaires was administered to a sample of 186 family members ( 93 primary and 93 secondary caregivers). The results indicate that primary caregivers presented worse scores in general health, anxiety, depression and psychopathological symptoms, compared with the group of secondary caregivers. The frequency of subjects (primary versus secondary caregivers) who reached the cut-off point in the SCL-90-R Global Severity Index (GSI) of psychopathology was similar for both groups. Even though the two caregiver groups presented a similar prevalence of psychopathology, the results suggest that mainly primary caregivers find themselves in a situation with risk for their general mental health, anxiety and depression.
\end{abstract}

Keywords: primary caregiver, eating disorders, caregiver experience, anxiety, depression, psychopathology.

Recibido: 25-mayo-2010; aceptado: 2-julio-2010

Correspondencia: Ana R. Sepúlveda, Facultad de Psicología, Universidad Autónoma de Madrid. Campus de Cantoblanco. 28049. Madrid. Correo-e: anarosa.sepulveda@uam.es

Agradecimientos: Este trabajo ha sido posible gracias a la concesión de una beca a la Dra. Sepúlveda del programa Ramón y Cajal del Ministerio de Ciencia e Innovación (RYC-2009-05092). Agradecemos la valiosa colaboración de la Asociación de padres ADANER-Madrid, como a todos los familiares que han participado en el estudio.

\section{INTRODUCCIÓN}

La experiencia de cuidar a una persona con una enfermedad grave y prolongada puede tener efectos muy negativos sobre la salud, tanto física como psíquica, en las personas que adquieren el rol como cuidador (Barrowclough, Tarrier, y Johnston M., 1996; Biegel, Milligan, Putnam, y Sung, 1994). Los cambios a nivel 
institucional han ido dirigidos a una mayor permanencia del paciente en su medio social y familiar, dejando a un lado el concepto de la institución como un lugar donde se satisfacen completamente las necesidades de los pacientes (Olabarría y Mansilla, 2007). La mayoría de los estudios que investigan el efecto de cuidar a una persona con una enfermedad mental, se han centrado en cuidadores y/o familiares de enfermos de Alzheimer o de esquizofrenia (Baronet, 1999; Barrowclough et al., 1996; Biegel et al., 1994; Cohen, 2000). Los resultados de estos estudios muestran que los efectos habituales son el estrés, la angustia, sentimientos de soledad, dependencia y pérdida, junto con dificultades en el funcionamiento familiar (Saunders, 2003; Schene, van Wijngaarden, y Koeter, 1998). Los cuidadores suelen tener peor salud que los restantes miembros de la familia que no están involucrados en los cuidados del enfermo y padecen mayores problemas orgánicos y fisiológicos, como patologías cardiovasculares o dolores osteomusculares (Schulz et al., 2002). Asimismo, el impacto de la enfermedad en el cuidador tiene a su vez, un efecto negativo en el paciente (p.ej. el número de comentarios críticos de los familiares es un factor pronóstico fiable de recaída del paciente con esquizofrenia) (Kuipers, 1992).

La anorexia nerviosa (AN) está considerada la tercera enfermedad crónica más frecuente de la adolescencia (Lucas, Beard, O'Fallon, y Kurland, 1991). Los tratamientos de los pacientes con trastornos de la conducta alimentaria (TCA) son prolongados y costosos, y un 20\% de los casos se cronifican (Morande, Celada, y Casas, 1999; Ratnasuriya, Eisler, Szmukler, y Russell, 1991). En contraste, estudios sobre los cuidadores de personas con trastornos del comportamiento alimentario han recibido menos atención científica aunque se han incrementado en esta última década (Highet, Thompson, y King, 2005; Kyriacou, Treasure, y Schmidt, 2008a; Kyriacou, Treasure, y Schmidt, 2008b; Monteleone, Treasure, Santonastaso, Favaro, y Brambilla, 2005; Sepulveda et al., 2009; Graap et al., 2008b; Winn et al., 2006). En un estudio sobre una amplia muestra de familias con un paciente psiquiátrico y de diferentes patologías (esquizofrenia, TCA, depresión mayor, trastor- no bipolar, trastorno de ansiedad, abuso de substancias y trastorno adaptativo), se observa el efecto indistinto de tener un familiar con un trastorno mental independientemente de la patología en fase aguda del trastorno o tras ser hospitalizado (Friedman y Mc Dermut, 1997). En cambio, otro estudio muestra que los cuidadores en trastornos alimentarios tienen mayores niveles de malestar y de dificultades en su rol de cuidador comparado con cuidadores de pacientes psicóticos (Treasure et al., 2001). Por otro parte, el estudio de Graap et al. (2008a) encontraron diferencias significativas respecto a las características y necesidades de los familiares pertenecientes a distintos subtipos de diagnóstico de trastorno alimentario. Se encontraron puntuaciones más elevadas en el malestar psicológico medido por el GHQ-12 en padres de pacientes con AN comparada con padres de pacientes con bulimia nerviosa (BN). A su vez, las madres informaron de mayores necesidades y problemas que los padres de forma significativa.Respecto a los factores que incrementan el malestar psicológico y dificultad personal en los familiares, destaca el miedo a la dependencia a largo plazo y la percepción de alto riesgo por las consecuencias de la enfermedad (Haigh y Treasure, 2003). En depresión por ejemplo, se ha constatado que el estilo explicativo negativo es un factor inespecífico de vulnerabilidad para experimentar malestar psicológico. En este sentido, Sanjuán, Pérez-García, Rueda y Ruiz (2008) han encontrado una mayor asociación de este estilo explicativo con los síntomas de ansiedad. Winn et al. (2006) encuentran otros factores, en familiares de pacientes de TCA, como el mayor número de horas de contacto con el paciente y valores altos en la emoción expresada. Otros estudios muestran que los familiares se acomodan a los rituales y conductas alimentarias nocivas como un «estilo de vida», adaptando su rutina y funcionamiento familiar a las exigencias de la patología (Sepúlveda et al., 2009).

Esta escasez de estudios sobre familiares se produce también en España, y por lo tanto, desconocemos el impacto de la enfermedad en la salud general y mental de los cuidadores, así como el impacto del estado de salud de los cuidadores sobre el paciente. Se ha evaluado el 
papel de la alexitimia en la familia (Espina, Ortego, Ochoa de Alda, y Alemán Méndez, 2001; Espina, 2003), la emoción expresada (Rodríguez y Vaz, 2005), depresión, ansiedad y ajuste marital (Ochoa de Alda, Espina, y Ortego, 2006) y un estudio de intervención familiar (Espina, Ortego, y Ochoa de Alda, 2003). Estos estudios muestran que los familiares obtienen peores puntuaciones en ansiedad, depresión, ajuste marital o salud general comparados con los grupos control (Ochoa de Alda et al., 2006). El estudio de Rodríguez y Vaz (2005) indicó que un $46,5 \%$ de las familias de pacientes con trastornos alimentarios presentaban niveles altos de emoción expresada, niveles más bajos que en familias de enfermos de esquizofrenia. Al igual que en otros estudios, las madres presentaban niveles más altos de sobre-implicación emocional que los padres.

No existe una definición unánimemente aceptada sobre lo que se denomina el cuidador principal. En la mayoría de los estudios se utiliza este término para referirse en general a uno o ambos padres (Moller, Gudde, Folden, y Linaker, 2009; Grov y Eklund, 2008) o bien se le equipara al término cuidador informal, refiriéndose a cualquier familiar o persona con un vínculo afectivo con el paciente. Algunos autores definen al cuidador principal, como la persona que atiende en primera instancia las necesidades físicas y emocionales de la persona enferma, que por lo general lo ejercen el/la esposo/a, hijo/a, o un familiar cercano al paciente (Astudillo y Mendinueta, 2008). En nuestro estudio hemos definido al cuidador principal (CP) según el tiempo semanal de contacto directo con el paciente, y quedaría definido como la persona que pasa más tiempo semanal en compañía del familiar con trastorno alimentario. En contraposición se definiría como cuidador secundario (CS), a la persona que pasa un menor tiempo de contacto personal. Esta definición ha sido utilizada previamente en varios estudios británicos (Sepulveda, Lopez, Todd, Whitaker, y Treasure, 2008; Whitney, Haigh, Weinman, y Treasure, 2007; Winn et al., 2006). En general, poco sabemos sobre la presencia de psicopatología en estos familiares y si a su vez, hay diferencias entre el tipo de cuidadores debido a la convivencia con pacientes que tienen una tendencia a la cronicidad y deterioro psicosocial.

Los objetivos del estudio fueron los siguientes: (1) examinar si hay diferencias en función de diversas variables sociodemográficas entre cuidadores principales versus secundarios en trastornos alimentarios, (2) evaluar si hay diferencias en el estado de salud general comparando entre tipo de cuidador y, (3) evaluar si hay diferencias en el nivel de psicopatología comparando entre tipo de cuidador.

\section{MÉTODO}

\section{Participantes}

La muestra estuvo constituida por un total de 186 familiares (51,6\% eran mujeres y 48,4\% eran varones) cuidadores de personas con un trastorno de la conducta alimentaria (TCA). La edad media de los participantes era de 47 años $(D T=5,1)$. El estudio fue trasversal y descriptivo a través de autoinforme. Los participantes fueron voluntarios, por lo que el muestreo fue no probabilístico (Hernández, Fernández, y Baptista, 2000). La muestra procedía de dos ámbitos, por un lado, de la asociación ADANER de Madrid y por otro, de dos unidades especializadas de trastornos alimentarios del Servicio de Psiquiatría. La distribución de la muestra fue la siguiente: un $8,6 \%(n=16)$ de la asociación ADANER, un $72 \%(n=134)$ del Hospital Niño Jesús en Madrid y un 19,4\% ( $n$ =36) del Hospital de Valdecilla en Santander. La muestra total fue dividida según la cantidad de horas de contacto con el paciente; un total de 93 fueron definidos como cuidadores principales (CP) y los restantes 93 fueron definidos como cuidadores secundarios (CS).

\section{Instrumentos}

Cuestionario socio-demográfico. Se solicitó información sociodemográfica de los curadores (nivel educativo, laboral, económico, antecedentes familiares), información relativa a los pacientes (tipo de trastorno, año de diagnostico, año de inicio de síntomas, presencia de sínto- 
mas específicos — vómitos o laxantes - y asociados - autolesiones, abuso de sustanciastipo y duración de los tratamientos, y numero de ingresos) así como datos acerca de la situación de cuidado (horas de contacto, y características de la relación de cuidado).

Cuestionario de Salud General (GHQ-12; Goldberg y Williams, 1988), en su versión española (Lobo y Muñoz, 1996). Una reciente validación con población general adulta española obtuvo una fiabilidad de 0,76 (SánchezLópez y Dresch, 2008). El GHQ-12 es un cuestionario que mide el estado general de salud. Fue diseñado para medir el cambio en la salud mental durante las dos semanas anteriores a su realización. La versión de doce ítems es la más reducida de las disponibles. Cada ítem se puntúa de 0 a 3 . El rango de puntuaciones va de 0 a 36, donde elevadas puntuaciones indican peor salud mental. La fiabilidad del cuestionario en muestra actual es adecuada con un alfa de Cronbach de 0,87 . El punto de corte en población general es $11 / 12$, que identifica población «caso» de malestar psicológico (Schmitz, Kruse, y Tress, 2001).

Escala Hospitalaria de Ansiedad y Depresión (HADS; (Zigmond y Snaith, 1983), traducida al castellano y validada en muestra clínica (Quintana et al., 2003). Esta escala consta de dos subescalas, ansiedad y depresión, donde cada una consta de 7 ítems, apareciendo de forma intercalada con 4 alternativas de respuestas que puntúan de 0 a 3 . La consistencia interna fue alta, con el mismo alfa de Cronbach de 0,86 para la escala de ansiedad como para la escala de depresión. Posteriormente fue validada en población general con similar fiabilidad (Terol et al., 2007). La fiabilidad del cuestionario en nuestro estudio se muestra muy similar a la de otros estudios tanto para la escala de Ansiedad $(\alpha=0,84)$ como para la escala Depresión $(\alpha=$ $0,85)$. Los criterios de clasificación son los siguientes: 0-7: ausencia de síntomas; 8-10: clínicamente límite o dudoso; 11-21: presencia de síntomas de ansiedad y/o depresión o caso.

Cuestionario SCL-90-R (Derogatis, 1977). Adaptado y validado en población general por González de Rivera (2002). Un total de 90 ítems evalúan el grado de malestar psicológico en pacientes psiquiátricos, como también en po- blación no clínica. Cada ítem se valora de acuerdo con una escala Likert de 5 puntos de 0 a 4. Se puede obtener una puntuación de 0 a 360 . La puntuación de cada dimensión es calculada como la media de los ítems que la componen. Consta de nueve dimensiones (Somatización, Obsesión-Compulsión, Sensibilidad Interpersonal, Depresión, Ansiedad, Hostilidad, Ansiedad Fóbica, Ideación Paranoide y Psicoticismo) y a partir de estas dimensiones se obtiene el Índice Global de Severidad (GSI; global symptom index) que indica el grado del malestar psicológico general. La consistencia interna de las distintas escalas del cuestionario tiene valores entre 0,81 y 0,90. El coeficiente alfa de Cronbach para toda la escala en la muestra total fue de 0,97. La versión española ofrece tres baremos según género: ausencia de morbilidad (población no clínica), presencia de morbilidad psiquiátrica menor (población psicosomática), y presencia de patología psiquiátrica (población psiquiátrica). Si la persona puntúa por encima en el centil 80 en el baremo de población no clínica (equivale a una puntuación típica de 58) en el GSI o al menos en dos dimensiones psicosomáticas, se pasa al baremo de población con alteración psicosomática; y si el GSI sobrepasa el centil 90 (equivale a una puntuación típica de 63, o corresponde a una puntuación directa de 0,80 en varones y 1,12 en mujeres) en el baremo de población no clínica, se pasa directamente al baremo psiquiátrico, y se considera con presencia de patología psiquiátrica.

\section{Procedimiento}

Para la recogida de información en la asociación ADANER, primero se envió una carta informativa sobre el estudio y se solicitó su colaboración. Posteriormente se mandó un segundo envío con la batería de cuestionarios a los socios que dieron su consentimiento $(10 \%$, $n=80$ ). El $100 \%$ de los que dieron su consentimiento completaron los cuestionarios y lo enviaron a la sede en Madrid por contra-reembolso. No conocemos los motivos de la baja participación por parte de los socios. El envío y la recogida de cuestionarios se realizaron desde octubre de 2006 a marzo de 2007. Para 
este estudio sólo se seleccionaron 16 padres, 8 padres y 8 madres que tenían en común la misma hija. En segundo lugar se obtuvo la colaboración de las unidades de trastornos alimentarios del Hospital Niño Jesús de Madrid y del Hospital Marqués de Valdecilla de Santander, en los que se entregó personalmente los cuestionarios a los familiares de pacientes de diferentes módulos de tratamiento y lo entregaron a la semana siguiente. El porcentaje en el Hospital Valdecilla de Santander fue del 100\% $(n=$ 53) de los cuestionarios dados durante dos meses. Para este estudio sólo se seleccionaron 36 padres, 18 padres y 18 madres que tenían en común la misma hija. La tasa de respuesta en el Hospital Niño Jesús se estima en alrededor de un $80 \%(n=176)$ de los cuestionarios. La duración del estudio fue desde enero 2008 a julio 2009. Para este estudio sólo se seleccionaron 134 padres (67 padres y 67 madres que tenían en común la misma hija). Los cuestionarios fueron respondidos exclusivamente por cuidadores de pacientes con TCA, por lo tanto, la información relativa a las pacientes utilizada en el estudio fue extraída a través de preguntas incluidas en el cuestionario sociodemográfico.

\section{Análisis estadístico}

Las variables continuas se describieron mediante índices de centralización y dispersión: media aritmética, desviación típica y rango. Las cualitativas se trataron analizando la frecuencia absoluta de cada una de las categorías. La normalidad de las variables continuas se verificó con el estadístico $Z$ de Kolmogorov-Smirnov. La comparación de los datos categóricos se realizó con la prueba $\chi^{2}$ o la corrección exacta de Fisher cuando fue pertinente. Las diferencias en las variables continuas en función de los grupos se contrastaron con la $t$ de Student para igualdad de medias en muestras dependientes. A su vez, se compararon los grupos según los puntos de corte en los cuestionarios GHQ, HADS y SCL-90-R para establecer la proporción de cuidadores de cada grupo que puntuaba en valores no-clínicos y clínicos. Para el análisis de los datos se utilizó el programa estadístico SPSS 15.0.

\section{RESULTADOS}

\section{Descripción sociodemográfica y clínica}

La distribución de los cuidadores principales y secundarios es del 50\%-50\% en la muestra, estando formado por 93 padres y 93 madres de la misma paciente. La Tabla 1 muestra las características sociodemográficas de los cuidadores y del familiar con TCA (datos informados por los cuidadores principales) y las diferencias según el tipo de cuidador. La mayor parte de los cuidadores conviven con la paciente. La edad media de los CP es mayor que la de los CS, y son en su mayoría mujeres. Encontramos diferencias estadísticamente significativas entre tipo de cuidador respecto al género, al nivel educativo, en la situación laboral, y horas de contacto.

La muestra total de pacientes con TCA $(n=$ 93) está compuesta principalmente por mujeres. La media de edad es 16,8 años. El diagnostico inicial más habitual es el de anorexia nerviosa; el resto de pacientes fue diagnosticado con bulimia nerviosa. Un 14,6\% $(n=18)$ no están en tratamiento. La duración media de la enfermedad es de 2 años y el tiempo medio que llevan en tratamiento de 12 meses.

\section{Diferencias en el estado de salud, ansiedad y depresión según tipo de cuidador}

Como podemos observar en la Tabla 2, existen diferencias estadísticamente significativas en el estado general de salud (GHQ-12) al comparar el grupo de CP con el grupo de CS. Los cuidadores principales obtuvieron una puntuación media mayor en comparación con los cuidadores secundarios. A su vez, los CP también obtuvieron puntuaciones más elevadas en los niveles de ansiedad y depresión (escala HADS) que los CS, de forma estadísticamente significativa $(p<0,05)$.

En la Figura 1 se muestran los porcentajes de sujetos con valores normales, moderados y clínicos para el malestar psicológico a través del GHQ-12 y los de ansiedad y depresión a través de las escalas del HADS. Utilizando un formato de puntuación continua en el GHQ-12, 
Tabla 1. Características sociodemográficas de los cuidadores y de las pacientes

\begin{tabular}{|c|c|c|c|c|}
\hline Cuidadores $(N=186)$ & $N$ & $\begin{array}{c}\text { Cuidadores } \\
\text { Principales } \\
(n=93)\end{array}$ & $\begin{array}{c}\text { Cuidadores } \\
\text { Secundarios (CS) } \\
\quad(n=93)\end{array}$ & \\
\hline Edad (años) & 186 & $\begin{array}{c}47,31(D T=5,1) \\
\text { Rango: } 37-61\end{array}$ & $\begin{array}{l}48,55(D T=7,1) \\
\text { Rango: } 19-62\end{array}$ & $\begin{array}{l}t(174)=-1,3 \\
\quad p=0,18\end{array}$ \\
\hline Genero & & & & \\
\hline $\begin{array}{l}\text { Hombre } \\
\text { Mujer }\end{array}$ & $\begin{array}{l}90 \\
96\end{array}$ & $\begin{array}{r}9,7 \% \\
90,3 \%\end{array}$ & $\begin{array}{l}87,1 \% \\
12,9 \%\end{array}$ & $\begin{array}{c}\chi^{2}(1)=111,6 \\
p<0,001\end{array}$ \\
\hline Nivel educación & & & & \\
\hline $\begin{array}{l}\text { Hasta Graduado Escolar } \\
\text { Bachillerato o superior }\end{array}$ & $\begin{array}{l}97 \\
83\end{array}$ & $\begin{array}{l}61,8 \% \\
38,2 \%\end{array}$ & $\begin{array}{l}46,2 \% \\
53,8 \%\end{array}$ & $\begin{array}{c}\chi^{2}(1)=4,43 \\
p=0,035\end{array}$ \\
\hline $\begin{array}{l}\text { Estatus marital } \\
\text { Casados / Viviendo juntos } \\
\text { Soltero/Divorciado/Viudo }\end{array}$ & $\begin{array}{c}167 \\
18\end{array}$ & $\begin{array}{r}91,4 \% \\
8,6 \%\end{array}$ & $\begin{array}{l}89,1 \% \\
10,9 \%\end{array}$ & $\begin{array}{c}\chi^{2}(1)=0,27 \\
p=0,60\end{array}$ \\
\hline $\begin{array}{l}\text { Situación laboral } \\
\text { Tiempo completo/parcial } \\
\text { Desempleado/Retirado }\end{array}$ & $\begin{array}{c}127 \\
59\end{array}$ & $\begin{array}{l}50,5 \% \\
49,5 \%\end{array}$ & $\begin{array}{l}86,0 \% \\
14,0 \%\end{array}$ & $\begin{array}{c}\chi^{2}(1)=27,0 \\
p<0,001\end{array}$ \\
\hline $\begin{array}{l}\text { Vive en la actualidad con la pacien } \\
\text { Si } \\
\text { No }\end{array}$ & $\begin{array}{c}169 \\
17\end{array}$ & $\begin{array}{r}95,7 \% \\
4,3 \%\end{array}$ & $\begin{array}{l}86,0 \% \\
14,0 \%\end{array}$ & $\begin{array}{c}\chi^{2}(1)=0,79 \\
p=0,007\end{array}$ \\
\hline $\begin{array}{l}\text { Horas de contacto } \\
\text { Menos de } 21 \mathrm{~h} / \mathrm{sem} \\
\text { Más de } 21 \mathrm{~h} / \mathrm{sem} \\
\end{array}$ & $\begin{array}{c}47 \\
134\end{array}$ & $\begin{array}{r}8,9 \% \\
91,1 \%\end{array}$ & $\begin{array}{l}42,9 \% \\
57,1 \%\end{array}$ & $\begin{array}{c}\chi^{2}(1)=27,15 \\
p<0,001\end{array}$ \\
\hline Pacientes $(N=93)$ & $N$ & Pacientes & & \\
\hline Edad media (años) & 93 & $\begin{array}{l}\text { 16,8 }(D T=3,4) \\
\text { Rango: } 12-30\end{array}$ & - & - \\
\hline $\begin{array}{l}\text { Género } \\
\text { Hombre } \\
\text { Mujer }\end{array}$ & $\begin{array}{c}5 \\
88\end{array}$ & $\begin{array}{l}5,4 \% \\
94,6 \%\end{array}$ & - & - \\
\hline $\begin{array}{l}\text { Diagnóstico } \\
\text { AN } \\
\text { BN }\end{array}$ & $\begin{array}{l}70 \\
23\end{array}$ & $\begin{array}{l}75,3 \% \\
24,7 \%\end{array}$ & - & - \\
\hline $\begin{array}{l}\text { Duración media de la enfermedad } \\
\text { (años) }\end{array}$ & 93 & $\begin{array}{l}2,2(D T=4,6) \\
\text { Rango }=0-12\end{array}$ & - & - \\
\hline $\begin{array}{l}\text { Edad de inicio de la enfermedad } \\
\text { (años) }\end{array}$ & 93 & $\begin{array}{l}14,45(D T=2,6) \\
\text { Rango: } 10-28\end{array}$ & - & - \\
\hline Edad de diagnóstico & 93 & $\begin{array}{l}15,0(D T=2,7) \\
\text { Rango: } 11-29\end{array}$ & - & - \\
\hline
\end{tabular}

67 cuidadores principales (77\%) y 56 cuidadores secundarios $(65 \%)$ superaron el punto de corte (11/12), que indica la presencia de malestar psicológico, sin que esta diferencia sea estadísticamente significativa, $\chi^{2}(1)=2.9, p=$ 0,08 .
Respecto a los puntos de corte en la subescala HADS-Ansiedad, el 33,3\% de la muestra de CP no tenía síntomas de ansiedad, el 32,3\% de la muestra de CP obtuvo valores clínicamente límite mientras que el 34,4\% presentó ansiedad clínica (puntuación $\geq 11$ ). Respecto a los cuida- 
dores secundarios (CS), un 35,9\% presentaban ausencia de ansiedad, un 22,8\% tenían niveles clínicamente límite y un $41,3 \%$ presentaban ansiedad clínica. No se encontraron diferencias estadísticamente significativas entre tipo de cuidadores, $\chi^{2}(2)=2,1$, n.s.

Respecto a los resultado de las subescala HADS-Depresión, el 39,6\% de la muestra de CP no presentó síntomas depresivos, en cambio el $19,8 \%$ de los CP puntuaron con valores clínicamente límite, mientras que el mismo número, $19,8 \%$ de los CP presentaron depresión clínica (puntuar $\geq 11$ ). De los CS, el $25 \%$ obtenían valores límites de depresión, mientras que el $19,6 \%$ presentaban valores de depresión clínica. Tampoco en este caso se hallaron diferencias estadísticamente significativas, $\chi^{2}(2)=7,5$, n.s.

\section{Diferencias en los niveles de psicopatología según el tipo de cuidador}

Las puntuaciones medias obtenidas en el SCL-90-R se presentan en la Tabla 2 para la muestra total en TCA, y entre cuidadores según género, ya que los baremos de comparación para muestra española se ofrecen según género (González de Rivera, 2002) y para la muestra general no clínica $(n=530)$ de la validación española. Al comparar las puntuaciones en las diferentes subescalas del SCL-90-R entre tipo de cuidadores, cuidadores principales $(n=84$ mujeres) versus cuidadores secundarios $(n=81$ varones), encontramos diferencias estadísticamente significativas en las siguientes escalas: escala de somatización, escala obsesivo-compulsivo, escala de depresión, escala de ansiedad, ansiedad fóbica y en el GSI (véase la Tabla 2). En todas ellas la puntuación de los cuidadores principales fue mayor que la de los cuidadores secundarios, indicando un peor estado en las distintas dimensiones psicopatológicas. A su vez, la muestra total de cuidadores en trastornos alimentarios presentaban puntuaciones superiores comparados con la muestra general no clínica de la validación española en la mayoría de las dimensiones psicopatológicas, excepto en las dimensiones de hostilidad y ideación paranoide (Tabla 2). La muestra total de cuidadores obtiene un GSI más elevado que la muestra general española.

Se pasa directamente al baremo psiquiátrico si la puntuación en el GSI supera el centil 90 en el baremo de población general española, que equivale a una puntuación típica de 63 o una puntuación directa de 0,80 en varones y de 1,12 en mujeres (Gónzalez de Rivera, 2002). Esta puntuación indica que existe un riesgo de alteraciones psicopatológicas o que cumple con criterios clínicos de malestar psicopatológico. El $13,97 \%(n<=13)$ de los CP y el $15,05 \%$ $(n=14)$ de los CS se sitúan por encima de estos

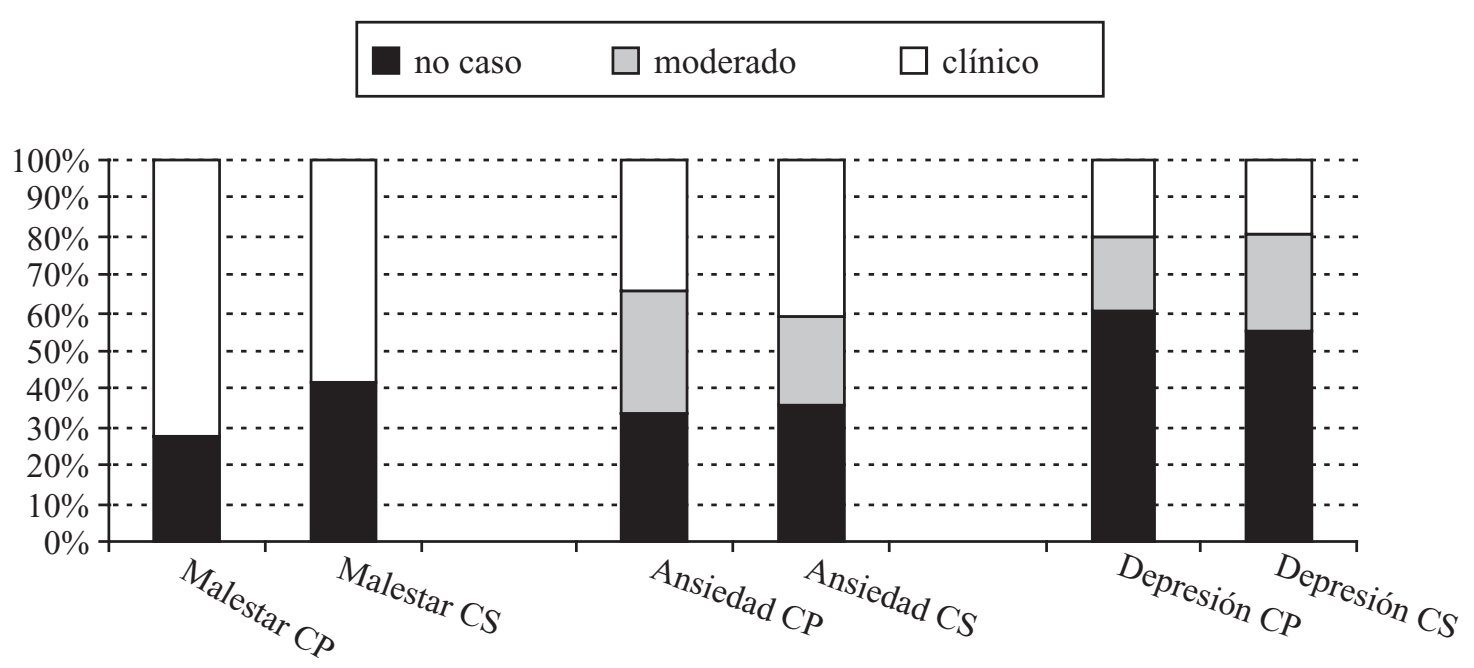

Figura 1. Comparativa de los valores de malestar psicológico, ansiedad y depresión en cuidadores principales (CP) y cuidadores secundarios (CS). 
Tabla 2. Medias y desviaciones típicas (entre paréntesis) en GHQ, HADS, y SCL-90-R para los cuidadores principales (CP) y cuidadores secundarios (CS), y para la población total de cuidadores. Se indican también las puntuaciones correspondientes a la población general de validación española del SCL-90-R

\begin{tabular}{lccccc}
\hline & $\begin{array}{c}\text { Población } \\
\text { general }\end{array}$ & $\begin{array}{c}\text { Muestra total } \\
\text { de cuidadores }\end{array}$ & $\begin{array}{c}\text { Cuidadores } \\
\text { principales }\end{array}$ & $\begin{array}{c}\text { Cuidadores } \\
\text { secundarios }\end{array}$ & CP vs. CS \\
& Media $(D T)$ & Media $(D T)$ & Media $(D T)$ & Media $(D T)$ & $t(126-181)$ \\
\hline GHQ-12 & - & $15,5(5,5)$ & $16,6(5,4)$ & $14,4(5,35)$ & $2,71^{* *}$ \\
HADS-Depresión & - & $7,1(4,14)$ & $8,0(4,2)$ & $6,25(3,9)$ & $2,87^{* *}$ \\
HADS-Ansiedad & - & $9,2(4,15)$ & $10,2(4,3)$ & $8,16(3,9)$ & $3,45^{* * *}$ \\
\hline SCL90-R & $N=530$ & $N=186$ & $n=93$ & $n=93$ & \\
\hline Somatización & $0,55(0,55)$ & $0,75(0,68)$ & $0,99(0,68)$ & $0,58(0,63)$ & $4,22^{* * *}$ \\
Obsesivo-compulsivo & $0,6(0,51)$ & $0,88(0,64)$ & $1,03(0,68)$ & $0,78(0,58)$ & $2,20^{*}$ \\
Sensibilidad Interoceptiva & $0,45(0,44)$ & $0,65(0,55)$ & $0,60(0,54)$ & $0,51(0,56)$ & 0,95 \\
Depresión & $0,72(0,55)$ & $1,16(0,74)$ & $1,39(0,73)$ & $0,94(0,71)$ & $3,42^{* * *}$ \\
Ansiedad & $0,52(0,49)$ & $0,72(0,67)$ & $0,90(0,71)$ & $0,60(0,60)$ & $2,62^{* *}$ \\
Hostilidad & $0,45(0,53)$ & $0,65(0,62)$ & $0,72(0,65)$ & $0,65(0,59)$ & 0,51 \\
Ansiedad Fóbica & $0,25(0,36)$ & $0,23(0,41)$ & $0,33(0,46)$ & $0,17(0,34)$ & $2,49 * *$ \\
Paranoidismo & $0,47(0,5)$ & $0,46(0,50)$ & $0,50(0,52)$ & $0,43(0,47)$ & 0,72 \\
Psicoticismo & $0,21(0,3)$ & $0,39(0,42)$ & $0,47(0,46)$ & $0,33(0,38)$ & 1,23 \\
GSI & $0,51(0,36)$ & $0,67(0,49)$ & $0,79(0,49)$ & $0,58(0,48)$ & $2,10^{* *}$ \\
\hline
\end{tabular}

Nota. GSI $=$ global severity index. ${ }^{a}$ González de Rivera (2002).

$* p<0,05 ; * *<<0,01 ; * * * p<0,001$.

baremos de puntuación. Dentro del grupo de cuidadores principales (el 10\% son varones y el $90 \%$ son mujeres), un $11 \%$ de varones $(n=$ $1 / 9)$ y un $23 \%(n=12 / 84)$ de mujeres superan el centil 90 y cumplen el criterio clínico de psicopatología $\left(\chi^{2}(1)=8,5, p=0,01\right)$. Dentro del grupo de cuidadores secundarios (87\% son varones y el $13 \%$ mujeres), el $20 \%$ de los varones $(n=12 / 81)$ y el $14 \%(n=2 / 12)$ de las mujeres cumplen criterios clínicos de malestar psicopatológico, de forma significativa $\left(\chi^{2}(1)\right.$ $=3,1, p=0,007$ ).

Centrándonos en la muestra de CP-mujeres $(n=12)$ que superan el centil $90 \mathrm{y}$ que pasa directamente a los baremos psiquiátricos, su media es de $1,55(D T=0,39)$, que equivale a una centil 45. La muestra de CS-varones $(n=12)$ que superan el centil 90 y que pasa directamente a los baremos psiquiátricos, su media es de 1,31 (DT $=0,55)$, que equivale a una centil 60 .

La Figura 2 muestra la comparación de puntuaciones medias de los cuidadores principales en trastornos alimentarios, mayoritariamente mujeres, con la muestra de población general femenina (Gónzalez de Rivera, 2002) y el punto de corte del Centil 80 del baremo de población general de cada una de las dimensiones del SCL-90-R y GSI. Los cuidadores principales ( $n$ $=84$ mujeres) superan la puntuación media de la muestra general femenina de cada dimensión psicopatológica. Respecto al punto de corte del centil 80, la puntuación media de la muestra de CP supera dos dimensiones, depresión y psicoticismo.

La Figura 3 muestra la comparación de puntuaciones medias de los cuidadores secundarios en trastornos alimentarios, mayoritariamente varones, con la muestra de población general masculina (Gónzalez de Rivera, 2002) y el punto de corte del Centil 80 del baremo de varones de población general de cada una de las dimensiones del SCL-90-R y GSI. Los cuidadores principales ( $n=81$ varones) superan la puntuación media de la muestra general masculina de cada dimensión psicopatológica, excepto ansiedad fóbica e ideación paranoica. Respecto al 


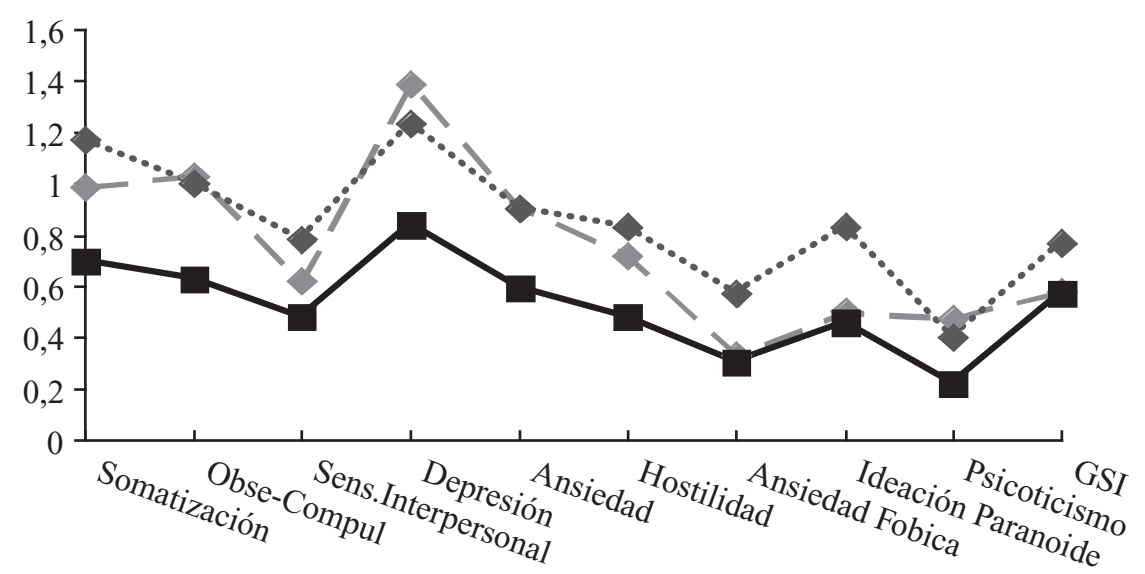

CUIDADOR PRINCIPAL: MUJERES $(n=84)$

Figura 2. Distribución de las puntuaciones medias de las mujeres cuidadores principales (CP) en las subescalas y GSI del SCL-90-R, comparadas con la muestra general no clínica (Gónzalez de Rivera, 2002), y punto de corte por encima del centil 80 del baremo de la muestra general.
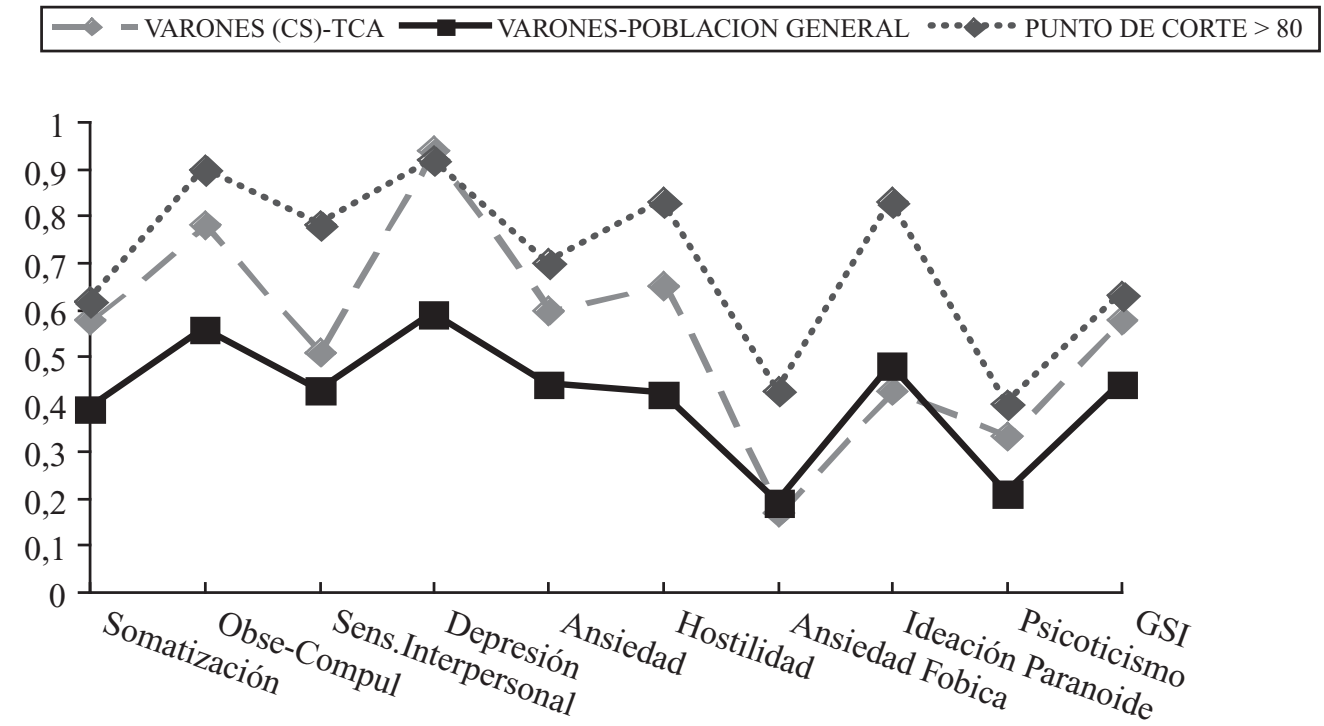

\section{CUIDADOR SECUNDARIO: VARONES $(n=81)$}

Figura 3. Distribución de las puntuaciones medias de los varones cuidadores secundarios (CS) en las subescalas y GSI del SCL-90-R, comparadas con la muestra general no clínica (Gónzalez de Rivera, 2002), y punto de corte por encima del centil 80 del baremo de la muestra general. 
punto de corte del centil 80, la puntuación media de la muestra de CS supera sólo la dimensión de depresión.

\section{Asociación entre variables psicológicas y sociodemográficas}

Dentro del grupo de cuidadores principales (CP) la puntuación total del GHQ-12 obtuvo una fuerte correlación positiva con las puntuaciones en la subescala HADS-Ansiedad $(r=$ $0,69 ; p<0,01)$, y con la subescala HADS-Depresión $(r=0,73 ; p<0,01)$; se asoció de forma moderada con el Índice Global de Severidad (GSI) $(r=0,51 ; p<0,01)$. La puntuación total en el GSI se asoció positivamente con el HADSAnsiedad $(r=0,68 ; p<0,01)$ y con el HADSDepresión $(r=0,61 ; p<0,01)$.

En el grupo de cuidadores secundarios (CS), la puntuación total del GHQ-12 obtuvo una correlación moderada y positiva con la subescala HADS-Ansiedad $(r=0,52 ; p<0,01)$ y con el Índice Global de Severidad (GSI) $(r=0,58$; $p<0,01)$, y una correlación más fuerte con la subescala de HADS-Depresión $(r=0,69 ; p<$ $0,01)$ La puntuación total en el GSI se asoció de forma más elevada con el HADS-Ansiedad $(r=0,74 ; p<0,01)$ y con el HADS-Depresión $(r=0,79 ; p<0,01)$.

En el grupo de cuidadores principales se encontró una correlación negativa entre la edad de los padres y la puntuación total en el GHQ$12(r=-0,24 ; p<0,05)$ y una correlación positiva entre la duración de la enfermedad y el GSI $(r=0,32 ; p<0,05)$. No se encontró ninguna asociación según tipo de diagnóstico de la paciente (AN vs. BN) $(p>0,05)$. No se hallaron asociaciones en el grupo de cuidadores secundarios $(p>0,05)$.

\section{DISCUSION}

El primer objetivo de este estudio era examinar si había diferencias sociodemográficas entre cuidadores principales y secundarios. Los resultados muestran la existencia de diferencias significativas en las variables sociodemográficas entre CP-CS. Los cuidadores principales fueron mayoritariamente mujeres y con un menor nivel educativo. Respecto a la situación laboral, sólo una mitad de los CP trabajaba parcial o a tiempo completo comparado con las tres cuartas partes de los cuidadores secundarios, situación que permite o es causa de que tengan un mayor número de horas de contacto de forma significativa con la paciente. La distribución de nuestra muestra en CP y CS ofrece una imagen de la situación de cuidador en la sociedad española, y nos ofrece un perfil del cuidador principal que concuerda con estudios realizados en otras poblaciones en salud mental (Astudillo et al., 2008; Garcia-Calvente, MateoRodriguez, y Eguiguren, 2004; Islas Salas, Ramos del Rio, Aguilar Estrada, y Garcia Guillén, 2006). En el cuidado de pacientes crónicos suelen ser las mujeres y las hijas las que se hacen cargo del rol como cuidador (Astudillo et al., 2008), y en el caso de pacientes con trastorno alimentario son las madres principalmente las encargadas de esta función, como podemos observar en nuestro estudio.

La comparación en el estado de salud entre CP-CS, que constituye el segundo objetivo de nuestro estudio, muestra que los valores totales de los distintos marcadores de salud relacionados con el malestar psicológico, ansiedad y depresión fueron significativamente más elevados en los CP que en los CS. Comparando la distribución del punto de corte en el GHQ-12 encontramos que el $77 \%$ de los CP y el $65 \%$ de los CS muestran elevado malestar psicológico. La comparación con otros estudios muestran que el valor de las puntuaciones en el GHQ-12 era más elevadas en el presente estudio, comparados con el estudio de Winn et al., (2006) donde el 50,9\% obtenían elevado malestar psicológico o el $59 \%$ de los cuidadores en el estudio de Haigh y Treasure (2003). Con respecto a los valores en el cuestionario HADS, los CS muestran una proporción ligeramente más alta de personas con valores clínicos en ansiedad (34,4\% de los CP vs. $41,3 \%$ de los CS), aunque no existen diferencias estadísticamente significativas y una proporción similar respecto a la depresión clínica $(19,8 \%$ de CP vs. $19,6 \%$ de CS). Estos datos podrían indicar que los cuidadores principales sufren un mayor impacto de la tarea de cuidado mostrando unos valores más 
altos en las puntuaciones medias de malestar psicológico, ansiedad y depresión, aunque esta diferencia entre tipos de cuidador desaparece cuando se examina la proporción de población de mayor riesgo clínico para cada dimensión. Es decir, que posiblemente, halla una transmisión de malestar psicológico, de ansiedad y depresión en paralelo en la familia, cuando uno de los cuidadores se encuentre en un alto riesgo clínico de padecer problemas de salud mental.

Recientemente, se ha revisado los estudios que comparan familiares en trastornos alimentarios con otros grupos de control, encontrándose sólo tres estudios que comparan los niveles de estrés, ansiedad y depresión de familias en TCA y otros grupos control (Zabala, Macdonald, y Treasure, 2009). Uno de ellos es el estudio de Kyriacou et al., (2008a), que comparó 151 cuidadores de TCA y 93 cuidadores de un familiar sano como grupo control y encontró que el $50 \%$ del primer grupo presentaban ansiedad clínica utilizando el HADS comparado con el 15\% del grupo control y $13 \%$ presentaba depresión clínica comparado con el 1\% del grupo control. En nuestra muestra, obtenemos un porcentaje menor respecto a la ansiedad clínica y ligeramente mayor en depresión clínica, tanto para los cuidadores principales como para los secundarios. En otro estudio se encontró que los cuidadores de pacientes con un TCA experimentaban niveles de malestar psicológico más altos en comparación con cuidadores de pacientes psicóticos (Treasure et al., 2001), indicando mayor morbilidad psicológica. En general, estos estudios apoyan la idea de que los cuidadores de pacientes de TCA presentan niveles altos o muy altos de malestar y problemas de salud respecto a otros grupos de comparación.

Estos resultados apoyarían el modelo de estrés y sufrimiento en familiares de TCA (Schmidt y Treasure, 2006; Treasure et al., 2007), en el cual las relaciones intra-familiares de interacción y conducta son relevantes. Según este modelo, las relaciones interpersonales contribuyen a la continuación de la enfermedad si están caracterizadas por un esfuerzo excesivo o insuficiente como respuesta a la enfermedad. Se sugiere que la respuesta emocional continua de los padres puede subyacer ciertos rasgos propios de personalidad tales como tendencias compulsivas o ansiosas, las cuáles a su vez, pueden ser reducidas con una apropiada intervención psicoeducativa. Por ejemplo, Sepúlveda y colaboradores (2009) muestra que los valores de ansiedad y depresión pueden ser modificados a través de una intervención familiar estructurada que incluye a cuidadores principales y secundarios, donde el $61,3 \%$ presentaba ansiedad clínica al inicio de la intervención y se redujo al $48 \%$, mientras del $20 \%$ presentaba depresión clínica y se redujo al $17 \%$ de forma significativa tras 3 meses de intervención, como también se mejoró la emoción expresada relacionada con la sobreimplicación y los aspectos negativos como cuidador (Sepulveda, Kyriacou, y Treasure, 2009).

A nivel de psicopatología, nuestro tercer objetivo del estudio, encontramos también diferencias significativas entre CP y CS. Los cuidadores principales presentan valores más altos en todas las subescalas del SCL-90-R y aparecen diferencias estadísticamente significativas en las dimensiones de somatización, obsesivocompulsivo, depresión, ansiedad, ansiedad fóbica y en el índice de severidad global. Al comparar la proporción de sujetos que superan el centil 90 del GSI y pasan por tanto al baremo psiquiátrico, CP y CS muestran unos valores similares $(13,97 \%$ y $15,05 \%$ respectivamente), lo cual indica una similar gravedad psicopatológica en ambos tipos de cuidadores. Los cuidadores principales en trastornos alimentarios, mayoritariamente mujeres, superan las puntuaciones medias de población general femenina (Gónzalez de Rivera, 2002) y superan el punto de corte del centil 80, en dos dimensiones, depresión y psicoticismo. Mientras los cuidadores secundarios, mayoritariamente varones, superan las puntuaciones medias de población general masculina excepto en hostilidad e ideación paranoica (Gónzalez de Rivera, 2002) y superan el punto de corte del centil 80, sólo en depresión. Estos datos indican un alto grado de malestar psicopatológico en ambas muestras de cuidadores. El uso del cuestionario SCL-90-R está indicado en la literatura como medida de malestar general, pese a que su uso como indicador clínico de psicopatología esta altamente discutido. Sin embargo, la evaluación de síntomas psicopatológicos es importante por las im- 
plicaciones que puede tener de cara al tratamiento, algo que es aceptado en, por ejemplo, el tratamiento de personas con adicciones (López, Fernández y Becoña, 2009).

La distribución de cuidadores femeninos y masculinos en la variable tipo de cuidador puede estar en la base de estas diferencias. Como hemos mencionado más arriba, se observa un predominio de mujeres entre los CP y de varones en el CS. Las diferencias de género en la respuesta y afrontamiento de la ansiedad y en la aparición de alteraciones del estado de ánimo, son factores a tener en cuenta y que pueden afectar a la respuesta de los sujetos a los cuestionarios empleados en el estudio. Más específicamente, en el estudio de Ortiz, Moral, Ciccotelli y del Barrio (2003), utilizando el SCL-90, el factor «género» sí arrojó diferencias a favor de las mujeres; las mujeres puntuaron significativamente más en todos los índices globales además de en la mayoría de las escalas, exceptuando: hostilidad, ansiedad fóbica y psicoticismo. En el estudio de González de Rivera (2002) citado en Ortiz et al. (2003), las mujeres puntuaron más alto en las subescalas del SCL90 de somatización, depresión, ansiedad fóbica, así como en el GSI. Estos datos sugieren que, las diferencias de género encontradas en nuestro grupo de cuidadores es probable que no sean inherentes al papel de cuidador y que el impacto en el malestar del cuidador parece ser diferente para los padres y las madres de un familiar con un TCA, sean la mayoría de los primeros CS y la mayoría de las segundas CP.

El estudio presenta algunas limitaciones como por ejemplo la de no poseer un grupo control con el que comparar los datos de la muestra. El tamaño de la muestra, pese a ser amplio no lo es suficientemente para poder establecer una generalización. Además, la muestra está limitada ya que la gran mayoría son padres de adolescentes, habiendo una pequeña proporción de familiares de adultos.

En conclusión, de este estudio se desprende en primer lugar que los cuidadores de pacientes de un TCA se encuentran en una situación negativa en su estado de salud general. Aunque no podemos establecer relaciones causales entre el cuidado de la paciente y los resultados obtenidos, sí podemos concluir que los cuidadores principales de estos pacientes presentan claros síntomas de ansiedad, depresión y malestar psicológico. En segundo lugar, los cuidadores principales que, en nuestro estudio, son los que pasan un mayor número de horas de contacto con la paciente, acusan los efectos de la tarea de cuidar de la persona con trastorno mental, con valores más altos de ansiedad y de depresión que los cuidadores secundarios. También los CP presentan niveles más elevados en la mayoría de las dimensiones psicopatológicas, aunque ambos superan los valores medios de la población general. Si compramos la población de mayor riesgo clínico, estas diferencias desaparecen y ambos cuidadores superan el punto de corte de gravedad clínica en depresión. Por tanto, el tratamiento de los TCA debe incluir necesariamente a ambos cuidadores y prestar una atención adecuada a su estado de salud general y mental. La atención especifica a posibles cuadros de ansiedad o depresión de los cuidadores es viable a través de un trabajo específico con ellos y con programas psicoeducativos en los que se puedan generar estrategias mejorar las habilidades y competencias de los cuidadores en su autocuidado.

\section{REFERENCIAS}

Astudillo, A. W. y Mendinueta, A. C. (2008). Necesidades de los cuidadores del paciente crónico. In Sociedad Vasca de Cuidados Paliativos (Ed.), Guia de recursos sanitarios y sociales en la fase final de la vida en Guipuzkoa (pp. 235-255). San Sebastian.

Baronet, A. (1999). Factors associated with caregiver burden in mental illness: A critical review of the research literature. Clinical Psychology Review, 19, 819841.

Barrowclough, C., Tarrier, N., y Johnston M. (1996). Distress, expressed emotion, and attributions in relatives of schizophrenia patients. Schizophrenia Bulletin, 22, 691-702.

Biegel, D. E., Milligan, S. E., Putnam, P. L., y Sung, L. (1994). Predictors of burden among lower socio-economic status caregivers of persons with chronic mental illness. Community Mental Health Journal, 30, 473494.

Cohen, D. (2000). Caregivers for persons with Alzheimer's disease. Current Psychiatry Reports, 2, 32-39.

Derogatis, L. (1977). SCL-90-R. Administration, scoring and procedures Manual 1 for revised version of the 
SCL-90. (Baltimore: John Hopkins University Press ed.).

Espina, A. (2003). Alexithymia in parents of daughters with eating disorders: Its relationships with psychopathological and personality variables. Journal of Psychosomatic Research, 55, 553-560.

Espina, A., Ortego, M. A., y Ochoa de Alda, I. (2003). Dyadic adjustment in parents of daughters with an eating disorder. European Eating Disorders Review, 11, 349-362.

Espina, A., Ortego, M. A., Ochoa de Alda, I., y Alemán Méndez, A. (2001). Alexitimia, familia y trastornos alimentarios. Anales de Psicologia, 17, 139-149.

Friedman, M. y Mc Dermut, W. (1997). Family functioning and mental illness: a comparison of psychiatric and nonclinical families. Family Process Journal, 36, $357-$ 367.

García-Calvente, M. M., Mateo-Rodríguez, I., y Eguiguren, A. P. (2004). [The system of informal caregiving as inequality]. Gac.Sanit., 18 Suppl 1, 132-139.

Goldberg, D. P. y Williams, P. (1988). A User's Guide to the General Health Questionnaire. Windsor: NFERNelson.

González de Rivera, J. (2002). Manual del Cuestionario de 90 Sintomas (SCL-90-R). Madrid: TEA Ediciones.

Graap, H., Bleich, S., Herbst, F., Trostmann, Y., Wancata, J., y de Zwaan, M. (2008a). The needs of carers of patients with anorexia and bulimia nervosa. Eur.Eat. Disord.Rev., 16, 21-29.

Graap, H., Bleich, S., Herbst, F., Trostmann, Y., Wancata, J., y de Zwaan, M. (2008b). The needs of carers of patients with anorexia and bulimia nervosa. European Eating Disorder Review, 16, 21-29.

Grov, E. K. y Eklund, M. L. (2008). Reactions of primary caregivers of frail older people and people with cancer in the palliative phase living at home. Journal Advanced Nursing, 63, 576-585.

Haigh, R. y Treasure, J. (2003). Investigating the needs of carers in the area of eating disorders: Development of the Carers' Needs Assessment Measure (CaNAM). European Eating Disorders Review, 11, 125-141.

Hernández, R., Fernández, C., y Baptista, P. (2000). Metodología de la investigación. México: Mc Graw Hill.

Highet, N., Thompson, M., y King, R. M. (2005). The Experience of Living with a Person with an Eating Disorder: The Impact on the Carers. Eating Disorders: The Journal of Treatment $y$ \& Prevention, 13, 327-344.

Islas Salas, N., Ramos del Río, B., Aguilar Estrada, M., y García Guillén, M. (2006). Perfil psicosocial del cuidador primario informal del paciente con EPOC. Revista del Instituto Nacional de Enfermedades Respiratorias, 19, 266-271.

Kuipers, L. (1992). Expressed Emotion in 1991. Social Psychiatry and Psychiatric Epidemiology, 27, 1-3.
Kyriacou, O., Treasure, J., y Schmidt, U. (2008a). Expressed Emotion in Eating Disorders assessed via self-report: An examination of factors associated with Expressed Emotion in carers of people with Anorexia Nervosa in comparison to control families. International Journal of Eating Disorders, 41, 37-46.

Kyriacou, O., Treasure, J., y Schmidt, U. (2008b). Understanding how parents cope with living with someone with anorexia nervosa: modelling the factors that are associated with carer distress. International Journal of Eating Disorders, 41, 233-242.

Lobo, A. y Muñoz, P. E. (1996). General Health Questionnaire. Guide for the use of the different validated versions in Spanish language [Cuestionario de Salud General GHQ. Guía para el usuario de las distintas versiones en lengua española validadas]. Barcelona: Masson.

López, A. Fernández, E., y Becoña, E. (2009). Comparación de las puntuaciones del scl-90-R entre personas con dependencia de la nicotina y personas con dependencia de la cocaína al inicio del tratamiento. Revista de Psicopatología y Psicología Clínica, 14, 17-23.

Lucas, A. R., Beard, C. M., O'Fallon, W. M., y Kurland, L. T. (1991). 50-year trends in the incidence of anorexia nervosa in Rochester, Minn.: a population-based study. American Journal Psychiatry, 148, 917-922.

Moller, T., Gudde, C. B., Folden, G. E., y Linaker, O. M. (2009). The experience of caring in relatives to patients with serious mental illness: gender differences, health and functioning. Scandinavian Journal of Caring Sciences, 23, 153-160.

Monteleone, P., Treasure, J., Santonastaso, P., Favaro, A., y Brambilla, F. (2005). Families of people with an eating disorder. In N.Sartorius, J. Leff, J. Lopez-Ibor, M. Maj, y A. Okasha (Eds.), Families and Mental Disorders, John Wiley \& Sons.

Morande, G., Celada, J., y Casas, J. J. (1999). Prevalence of eating disorders in a Spanish school-age population. Journal Adolescent Health, 24, 212-219.

Olabarría, B. y Mansilla, F. (2007). Ante el burnout: Cuidados a los equipos de salud mental. Revista de Psicopatología y Psicología Clínica, 12, 1-14.

Ochoa de Alda, I., Espina, A., y Ortego, M. A. (2006). Un estudio sobre personalidad, ansiedad y depresión en padres de hijas con un trastorno alimentario. Clinica y Salud, 17, 151-170.

Quintana, J. M., Padierna, A., Esteban, C., Arostegui, I., Bilbao, A., y Ruiz, I. (2003). Evaluation of the psychometric characteristics of the Spanish version of the Hospital Anxiety and Depression Scale. Acta Psychiatrica Scandinavica, 107, 216-221.

Ratnasuriya, R. H., Eisler, I., Szmukler, G. I., y Russell, G. F. (1991). Anorexia nervosa: outcome and prognostic factors after 20 years. British Journal of Psychiatry, $158,495-502$. 
Rodríguez, L. y Vaz, F. J. (2005). Assessment of expressed emotion in families of patients with eating disorders: using the Camberwell Family Interview on a Spanish sample. Actas Españolas de Psiquiatría, 33, 359-365.

Sánchez-López, M. P. y Dresch, V. (2008). The 12-Item General Health Questionnaire (GHQ-12): reliability, external validity and factor structure in the Spanish population. Psicothema, 20, 839-843.

Sanjuán, P. Pérez-García, A.M., Rueda, B., y Ruiz, M.A. (2008). Estilos explicativos y afecto negativo. Revista de Psicopatología y Psicología Clínica, 13, 45-52.

Saunders, J. C. (2003). Families living with severe mental illness: A literature review. Issues in Mental Health Nursing, 24, 175-198.

Schene, A. H., van Wijngaarden, B., y Koeter, M. W. J. (1998). Family Caregiving in Schizophrenia: Domains and Distress. Schizoprenia Bulletin, 24, 609-618.

Schmidt, U. y Treasure, J. (2006). Anorexia Nervosa: Valued and Visible. A Cognitive-Interpersonal Maintenance Model and its Implications for Research and Practice. British Journal of Clinical Psychology, 45, $1-25$.

Schmitz, N., Kruse, J., y Tress, W. (2001). Improving screening for mental disorders in the primary care setting by combining the GHQ-12 and SCL-90-R subscales. Comprehensive Psychiatry, 42, 166-173.

Schulz, R., O’Brien, A., Czaja, S., Ory, M., Norris, R., Martire, L. M. et al. (2002). Dementia caregiver intervention research: in search of clinical significance. Gerontologist, 42, 589-602.

Sepúlveda, A. R., Kyriacou, O., y Treasure, J. (2009). Development and Validation of the Accommodation and Enabling Scale for Eating Disorders (AESED) for Caregivers in Eating Disorders. BMC Health Services Research, 9, 171-doi:10.1186/1472-6963-9-171.

Sepúlveda, A. R., López, C., Todd, G., Whitaker, W., y Treasure, J. (2008). An Examination of the Impact of Maudsley Eating Disorder Collaborative Care Workshops on the Well-Being of Family Members: A Pilot Study. Social Psychiatry and Psychiatric Epidemiology, 43, 584-591.
Sepúlveda, A. R., Todd, G., Whitaker, W., Grover, M., Stahl, D., y Treasure, J. (2009). Espressed emotion in relatives of patients with eating disorders following a skills training programme. International Journal of Eating Disorders, 42, 0-00.

SPSS (2006). SPSS; Version 15.0. Computer software. Chicago, Illinois: SPSS Inc.

Terol, M. C., López-Roig, S., Rodríguez-Marín, J., MartínAragón, M., Pastor, M. A., y Reig, M. (2007). Propiedades psicométricas de la escala hospitalaria de ansiedad y depresión (HADS) en población española. Ansiedad y Estrés, 13, 163-176.

Treasure, J., Murphy, T., Szmukler, G., Todd, G., Gavan, K., y Joyce, J. (2001). The experience of caregiving for severe mental illness: A comparison between anorexia nervosa and psychosis. Social Psychiatry y Psychiatric Epidemiology, 36, 343-347.

Treasure, J., Sepúlveda, A. R., Whitaker, W., Todd, G., López, C., y Whitney, J. (2007). Collaborative care between professionals and non professional in the management of eating disorders:a description of workshops focused on interpersonal maintaining factors. European Eating Disorders Review, 15, 24-34.

Whitney, J., Haigh, R., Weinman, J., y Treasure, J. (2007). Caring for people with Eating Disorders: Factors associated with psychological distress and negative caregiving appraisals in carers of people with eating disorders. British Journal of Clinical Psychology, 46, 413-428.

Winn, S., Perkins, S., Walwyn, R., Schmidt, U., Eisler, I., Treasure, J. et al. (2006). Predictors of mental health problems and negative caregiving experiences in carers of adolescents with bulimia nervosa. International Journal of Eating Disorders, 40, 171-178.

Zabala, M., Macdonald, P., y Treasure, J. (2009). Appraisal of Caregiving Burden, Expressed Emotion and Psychological Distress in Families of People with Eating Disorders: a Systematic Review. European Eating Disorders Review, 17, 338-349.

Zigmond, A. S. y Snaith, R. P. (1983). The hospital anxiety and depression scale. Acta Psychiatrica Scandinavica, 67, 361-370. 